\title{
The Electromagnetic Field in the Relativistic Uniform Model
}

\author{
Sergey G. Fedosin \\ PO box 614088, Sviazeva str. 22-79, Perm, Perm Krai, Russia \\ fedosin@hotmail.com \\ Received date: 04.06.2018, Accepted date: 28.11.2018
}

\begin{abstract}
The potentials and the field strengths of the electromagnetic field, the energies of particles and of the field are calculated for the relativistic uniformly charged system with invariant charge density. The difference between the relativistic approach and the classical uniform model is shown. The conclusion is proved that in the absence of the general magnetic field the energy of particles, associated with the scalar field potential, is twice as large in the absolute value as the energy, determined with the help of the tensor invariant of the electromagnetic field, which is part of the system's Hamiltonian.
\end{abstract}

Keywords: electromagnetic field, energy, relativistic uniform system

\section{Göreli Uniform Modelde Elektromanyetik Alan}

Öz

Elektromanyetik alanın potansiyelleri ve alan kuvvetleri, parçacıkların ve alanın enerjileri, değişmez yük yoğunluğuna sahip göreceli olarak uniform yüklü sistem için hesaplanır. Rölativistik yaklaşım ile klasik uniform modeli arasındaki fark gösterilmektedir. Sonuç olarak, genel manyetik alanın yokluğunda, skaler alan potansiyeline bağlı parçacıkların enerjisinin, Hamiltonian sistemin bir parçası olan elektromanyetik alanın tensör değişmezinin yardımıyla belirlenen enerji kadar mutlak değerde iki kat daha fazla olduğu sonucuna varılmıştır.

Anahtar Kelimeler: elektromanyetik alan, enerji, rölativistik uniform sistem

\section{INTRODUCTION}

In classical physics, the ideal uniform model of a body is widely used, in which the mass density is constant over the entire volume of the body or is given as the volume-averaged quantity. This model simplifies the solution of physical problems and allows us to quickly estimate various physical quantities. For example, the body mass is calculated simply by multiplying the mass density by the body volume, which is easier than integrating the density over the volume in case of the density's dependence on the coordinates. The disadvantage of the classical model is that the majority of real physical systems are far from this ideal uniformity.

The use of the relativistic uniform system's concept is based on the special theory of relativity and it is the next step towards more precise description of physical systems. In the relativistic approach the invariant charge density (and the invariant mass density) of the particles that make up the system is used. Due to the motion of particles, the effective charge density and mass density in the system differ from the invariant values, which introduces additional corrections to the values of the field functions and the system's energy.

Previously, the properties of the relativistic uniform system were studied in (Fedosin, 2014a; Fedosin, 2015; Fedosin, 2016a). The purpose of this work is to obtain more precise results in respect of the electromagnetic field, to calculate the second order corrections, as well as to check the relationship between the contributions into the relativistic energy of the system from the energy of particles in the scalar electric potential and from the proper energy of the electric field. The obtained results can be used to assess the properties of such relativistic objects as the proton and the charged neutron star corresponding to it. These objects are uniform enough, as the central mass density in them is only 1.5 times greater than the average density (Fedosin, 2012a; Fedosin, 2016a). We assume that distribution of the effective density of the electric charge over the volume of these objects is similar to distribution of the effective mass density, which is confirmed for a 
proton in (Kelly, 2001; Yakhshiev and Kim, 2013). In this case, we can assume that emerging of the radial dependence of the effective density is mainly associated with the radial dependence of the particles' speed, with almost constant invariant density of mass and charge. As a result, the use of the relativistic uniform model is quite reasonable, at least as a first approximation.

On the other hand, the theoretical approach we used would not be effective in respect of the objects, in which the electromagnetic forces between the charges were comparable in magnitude with the gravitational forces. In this case, due to the charges' repulsion from each other we can expect the surface distribution of the charge rather than uniform distribution over the volume. However, in neutron stars and protons there are additional forces, besides gravitation, which hold their matter and prevent the charge transfer. In the case of neutron stars it is strong interaction between closely spaced nucleons, which significantly increases the binding energy.

In the following sections, by solving equations of electromagnetic field inside and outside a stationary relativistic uniform system, the main field characteristics will be determined, including dependences of the electric potential and field strength on the current radius, as well as contribution to the system energy from the energy of charged particles in electric field and contribution from the integral tensor invariant. Then these energies will be compared with the electrostatic energy of the system and with the energy calculated using energymomentum tensor of the electromagnetic field.

\section{POTENTIALS AND FIELD STRENGTHS}

It is convenient to consider as a relativistic uniform system the spherical system consisting of charged particles. The system's stability can be maintained by gravitation, the internal pressure field and the particles' acceleration field (Fedosin, 2014b; Fedosin, 2016b). Moreover, the acceleration field tracks the motion of the particles kinematically rather than dynamically. It allows us to uniquely calculate the energy and the four-acceleration of the particles using the stress-energy tensor of the acceleration field, in contrast to many other variants of the stressenergy tensors of matter. In (Fedosin, 2016c) it was shown that due to the acceleration field an additional acceleration emerges in the system, which counteracts the gravitation force and changes the ratio of energies in the virial theorem. The equilibrium condition of the system in question follows from the equation of motion, presented in (Fedosin, 2016b) in the general form and in (Fedosin, 2016c) for typical particles, each of which occupies a representative volume element and determines the basic properties of the system. The wave equations hold true for the potentials of all the four fields, while at zero matter density in the solution for the pressure field and for the acceleration field the potentials of these fields vanish. For the field strengths and solenoidal vectors equations are used, the structure of which coincides with that of Maxwell equations.

The field functions are calculated on the assumption that there is no common rotation of particles in the system and at each point they move randomly. This leads to the absence of the mass currents and electric currents inside the system and to vanishing of the global vector potentials and the solenoidal vectors of all the fields. Indeed, from the solution of wave equations for a single moving charged particle it follows that the vector potential of the electromagnetic field of the particle is directed along its velocity. If we take the volume of any part of the system, containing a sufficiently large number of particles, and sum up the vector potentials of these particles, then, due to multidirectionality of the particles' velocities, the global vector potential in each of these volumes will tend to zero. The same can be said in respect of the magnetic field, which is calculated as the curl of the global vector potential. The magnetic field outside the system under consideration also turns out to be zero. Similarly, we can consider other fields that we use, including their global vector potentials and solenoidal vectors.

In principle, we can arrive at the conclusion that the vector potential of a particle is directed along its velocity without solving field equations. To do this, it is sufficient to take into account the general definition of the four-potential of the vector field for solid uniform particle (Fedosin, 2014b). On the other hand, while solving the equations we use the Lorentz gauge, which relates the partial derivative of the scalar potential with respect to time and the divergence of the vector potential. Due to the field's stationarity, the scalar potential does not depend on the time, and then the divergence of the vector potential must be zero, and the lines of the vector potential must be closed. Since the random motion of the particles does not allow these lines to be closed, 
the vector potential, averaged with respect to a certain volume of the system, becomes equal to zero.

The inhomogeneous electromagnetic wave equation for the scalar potential inside the sphere has the standard form:

$$
\Delta \varphi_{i}=-\frac{\rho_{0 q} \gamma^{\prime}}{\varepsilon_{0}}
$$

In Equation (1) the Lorentz factor of typical particles is $\gamma^{\prime}=\frac{1}{\sqrt{1-v^{\prime 2} / c^{2}}}, v^{\prime}$ is the root-meansquare speed of motion of an arbitrary particle inside the sphere, $c$ is the speed of light, $\varepsilon_{0}$ is the vacuum permittivity, $\rho_{0 q}$ is the particle's charge density in the reference frame associated with the particle, the index $i$ distinguishes the internal potential $\varphi_{i}$ from the external potential $\varphi_{o}$, which is generated by the sphere outside its limits. The value $\rho_{q}=\rho_{0 q} \gamma^{\prime}$ in Equation (1) represents the effective charge density. Both the potential $\varphi_{i}$ and $\gamma^{\prime}$ are the functions of the current radius $r$ inside the sphere and they do not depend on the angular variables or the time due to the stationarity of all the fields. Therefore, in the spherical coordinates, in the Laplacian it is enough to take only the part which depends on the radius:

$$
\frac{1}{r^{2}} \frac{d}{d r}\left(r^{2} \frac{d \varphi_{i}}{d r}\right)=-\frac{\rho_{0 q} \gamma^{\prime}}{\varepsilon_{0}}
$$

If we make substitution of variables in the form $\varphi_{i}=\frac{Z}{r}$, then the equation can be rewritten as follows:

$$
\frac{1}{r} \frac{d^{2} Z}{d r^{2}}=-\frac{\rho_{0 q} \gamma^{\prime}}{\varepsilon_{0}}
$$

The dependence of $\gamma^{\prime}$ on the radius was calculated in (Fedosin, 2015):

$$
\gamma^{\prime}=\frac{c \gamma_{c}}{r \sqrt{4 \pi \eta \rho_{0}}} \sin \left(\frac{r}{c} \sqrt{4 \pi \eta \rho_{0}}\right) \approx \gamma_{c}-\frac{2 \pi \eta \rho_{0} r^{2} \gamma_{c}}{3 c^{2}}
$$

where $\gamma_{c}$ is Lorentz factor of the particles at the center of the sphere, $\eta$ is the acceleration field coefficient, $\rho_{0}$ is the invariant mass density of the particles.

Substituting Equation (2) into the equation for $Z$, we have:

$\frac{d^{2} Z}{d r^{2}}=-\frac{\rho_{0 q} c \gamma_{c}}{\varepsilon_{0} \sqrt{4 \pi \eta \rho_{0}}} \sin \left(\frac{r}{c} \sqrt{4 \pi \eta \rho_{0}}\right)$

The general solution of this equation has the form:

$Z=C_{1}+C_{2} r+\frac{\rho_{0 q} c^{3} \gamma_{c}}{4 \pi \varepsilon_{0} \eta \rho_{0} \sqrt{4 \pi \eta \rho_{0}}} \sin \left(\frac{r}{c} \sqrt{4 \pi \eta \rho_{0}}\right)$

Since $\varphi_{i}=\frac{Z}{r}$, and in the center at $r=0$ the potential cannot be infinite, the coefficient $C_{1}$ must be equal to zero. Hence, the potential inside the sphere will equal:

$\varphi_{i}=C_{2}+\frac{\rho_{0 q} c^{3} \gamma_{c}}{4 \pi \varepsilon_{0} \eta \rho_{0} r \sqrt{4 \pi \eta \rho_{0}}} \sin \left(\frac{r}{c} \sqrt{4 \pi \eta \rho_{0}}\right)$

Let us now pass on to calculation of the external electric potential $\varphi_{o}$ of the fixed sphere, filled with moving charged particles. First, we will find the strength of the external electric field of the sphere. The Maxwell equations of the electromagnetic field have the standard form:

$$
\begin{aligned}
& \nabla \cdot \mathbf{E}=\frac{\rho_{0 q} \gamma^{\prime}}{\varepsilon_{0}} \quad \nabla \times \mathbf{B}=\frac{1}{c^{2}} \frac{\partial \mathbf{E}}{\partial t}+\frac{\rho_{0 q} \gamma^{\prime} \mathbf{v}^{\prime}}{\varepsilon_{0} c^{2}} \\
& \nabla \cdot \mathbf{B}=0 \quad \nabla \times \mathbf{E}=-\frac{\partial \mathbf{B}}{\partial t}
\end{aligned}
$$

According to Equation (4), the particles moving inside the sphere at velocities $\mathbf{v}^{\prime}$ generate around themselves the electromagnetic field with the strength $\mathbf{E}$ and the magnetic field $\mathbf{B}$. Let us surround the sphere with the shell of a spherical shape with an arbitrary radius $r \geq a$ and integrate the first relation in Equation (4) over the volume of the 
shell. We will also apply the Gauss theorem, replacing the integral of the divergence $\nabla \cdot \mathbf{E}$ with the integral of the vector $\mathbf{E}$ over the surface $S$ of the shell. Due to the symmetry of the sphere at the constant density $\rho_{0 q}$, for the vector $\mathbf{E}$ outside the sphere we find the following:

$$
\begin{gathered}
\int \nabla \cdot \mathbf{E}_{o} d V=\int \mathbf{E}_{o} \cdot \mathbf{n} d S=4 \pi r^{2} E_{o}=\frac{\rho_{0 q}}{\varepsilon_{0}} \int \gamma^{\prime} d V_{s} \\
\mathbf{E}_{o}=\frac{\rho_{0 q} c^{2} \gamma_{c} \mathbf{r}}{4 \pi \varepsilon_{0} \eta \rho_{0} r^{3}}\left[\frac{c}{\sqrt{4 \pi \eta \rho_{0}}} \sin \left(\frac{a}{c} \sqrt{4 \pi \eta \rho_{0}}\right)-a \cos \left(\frac{a}{c} \sqrt{4 \pi \eta \rho_{0}}\right)\right] \\
\mathbf{E}_{o}=\frac{q_{b} \mathbf{r}}{4 \pi \varepsilon_{0} r^{3}} \approx \frac{q \gamma_{c} \mathbf{r}}{4 \pi \varepsilon_{0} r^{3}}\left(1-\frac{3 \eta m}{10 a c^{2}}\right)
\end{gathered}
$$

the sphere: $q=\frac{4 \pi a^{3} \rho_{0 q}}{3}$. However, actually the electric field outside the sphere is defined by the charge $q_{b}$, which equals according to Equations (5$6)$ :

$$
\begin{aligned}
& q_{b}=\rho_{0 q} \int \gamma^{\prime} d V_{s}=\frac{\rho_{0 q} c^{2} \gamma_{c}}{\eta \rho_{0}}\left[\frac{c}{\sqrt{4 \pi \eta \rho_{0}}} \sin \left(\frac{a}{c} \sqrt{4 \pi \eta \rho_{0}}\right)-a \cos \left(\frac{a}{c} \sqrt{4 \pi \eta \rho_{0}}\right)\right] \approx \\
& \approx q \gamma_{c}\left(1-\frac{3 \eta m}{10 a c^{2}}\right)
\end{aligned}
$$

The relationship between the vectors of the electromagnetic field and the four-potential is the following:

$$
\partial_{0} A_{j}-\partial_{j} A_{0}=\frac{1}{c} E_{j} \quad \partial_{i} A_{j}-\partial_{j} A_{i}=-B_{k}
$$

where the indices $i, j, k=1,2,3$ do not coincide with each other.

$$
\begin{gathered}
\varphi_{o}=-\int E_{o} d r=\frac{\rho_{0 q} c^{2} \gamma_{c}}{4 \pi \varepsilon_{0} \eta \rho_{0} r}\left[\frac{c}{\sqrt{4 \pi \eta \rho_{0}}} \sin \left(\frac{a}{c} \sqrt{4 \pi \eta \rho_{0}}\right)-a \cos \left(\frac{a}{c} \sqrt{4 \pi \eta \rho_{0}}\right)\right] \\
\varphi_{o}=\frac{q_{b}}{4 \pi \varepsilon_{0} r} \approx \frac{q \gamma_{c}}{4 \pi \varepsilon_{0} r}\left(1-\frac{3 \eta m}{10 a c^{2}}\right)
\end{gathered}
$$

The space components $A_{j}$ of the four-potential are the components of the vector potential $\mathbf{A}$, which in this case is equal to zero. Consequently, in Equation (7) the components $E_{j}$ of the vector $\mathbf{E}$ are associated only with the time component $A_{0}=\frac{\varphi}{c}$ of the four-potential: $E_{j}=-\partial_{j} \varphi$. This equality in vector notation is written as follows: $\mathbf{E}=-\nabla \varphi$. Hence, in view of Equation (6), for $\mathbf{E}_{o}$ it follows that: 
At infinity, this potential becomes equal to zero. At the surface of the sphere potential in Equation (8) must coincide with the internal potential in Equation (3). Let us assume that $r=a$ and equate the two potentials. This allows us to determine the coefficient $C_{2}$ and to specify the internal potential $\varphi_{i}:$

$$
C_{2}=-\frac{\rho_{0 q} c^{2} \gamma_{c}}{4 \pi \varepsilon_{0} \eta \rho_{0}} \cos \left(\frac{a}{c} \sqrt{4 \pi \eta \rho_{0}}\right)
$$

$$
\varphi_{i}=\frac{\rho_{0 q} c^{2} \gamma_{c}}{4 \pi \varepsilon_{0} \eta \rho_{0} r}\left[\frac{c}{\sqrt{4 \pi \eta \rho_{0}}} \sin \left(\frac{r}{c} \sqrt{4 \pi \eta \rho_{0}}\right)-r \cos \left(\frac{a}{c} \sqrt{4 \pi \eta \rho_{0}}\right)\right] \approx \frac{\rho_{0 q} \gamma_{c}\left(3 a^{2}-r^{2}\right)}{6 \varepsilon_{0}}
$$

Now we can calculate the electric field strength inside the sphere. Taking into account the equality of the vector potential $\mathbf{A}$ to zero, we have the following:

$$
\begin{aligned}
& \mathbf{E}_{i}=-\nabla \varphi_{i}=\frac{\rho_{0 q} c^{2} \gamma_{c} \mathbf{r}}{4 \pi \varepsilon_{0} \eta \rho_{0} r^{3}}\left[\frac{c}{\sqrt{4 \pi \eta \rho_{0}}} \sin \left(\frac{r}{c} \sqrt{4 \pi \eta \rho_{0}}\right)-r \cos \left(\frac{r}{c} \sqrt{4 \pi \eta \rho_{0}}\right)\right] \approx \\
& \approx \frac{\rho_{0 q} \gamma_{c} \mathbf{r}}{3 \varepsilon_{0}}\left(1-\frac{2 \pi \eta \rho_{0} r^{2}}{5 c^{2}}\right)
\end{aligned}
$$

In Equation (10) we added the following expansion term, which contained the squared speed of light in the denominator and showed the difference of the relativistic uniform system from the classical case.

\section{ENERGY OF PARTICLES AND FIELDS}

$\int \rho_{0 q} \varphi_{i} \gamma^{\prime} d V=$

$=-\frac{\rho_{0 q}^{2} c^{4} \gamma_{c}^{2}}{4 \pi \varepsilon_{0} \eta^{2} \rho_{0}^{2}}\left\{\cos \left(\frac{a}{c} \sqrt{4 \pi \eta \rho_{0}}\right)\left[\begin{array}{l}\frac{c}{\sqrt{4 \pi \eta \rho_{0}}} \sin \left(\frac{a}{c} \sqrt{4 \pi \eta \rho_{0}}\right)- \\ -a \cos \left(\frac{a}{c} \sqrt{4 \pi \eta \rho_{0}}\right)\end{array}\right]-\frac{a}{2}+\frac{c}{4 \sqrt{4 \pi \eta \rho_{0}}} \sin \left(\frac{2 a}{c} \sqrt{4 \pi \eta \rho_{0}}\right)\right\} \approx$ $\approx \frac{3 q^{2} \gamma_{c}^{2}}{10 \pi \varepsilon_{0} a}\left(1-\frac{4 \eta m}{7 a c^{2}}\right)$

Note that the value of the energy obtained in Equation (11) is twice as large as the electrostatic energy of the charged particles inside the sphere. We will now calculate the integral over the volume of the tensor invariant of the electromagnetic field, separately for the field inside and outside the sphere. The integral of the tensor invariant is expressed in terms of the electric field strength and the magnetic field:
We will first calculate the contribution into the system's relativistic energy from the energy of particles in the electric field, which was defined in (Fedosin, 2016b) as the integral over the volume taken with respect to the product of the effective charge density inside the sphere $\rho_{q}=\rho_{0 q} \gamma^{\prime}$ by the internal scalar potential $\varphi_{i}$. In view of Equations (2) and (9), we have: $\int \frac{c^{2} \varepsilon_{0}}{4} F_{\mu \nu} F^{\mu v} d V=-\frac{\varepsilon_{0}}{2} \int\left(E^{2}-c^{2} B^{2}\right) d V$

This integral is included in this form in the relativistic energy of the system, where it makes the contribution from the electromagnetic field (Fedosin, 2016b). Substituting here Equations (10) and (6), and taking into account that $\mathbf{B}=0$, we find: 


$$
\begin{aligned}
& \int_{r=0}^{a} \frac{c^{2} \varepsilon_{0}}{4} F_{\mu \nu} F^{\mu v} d V=-\frac{\varepsilon_{0}}{2} \int_{r=0}^{a} E_{i}^{2} d V= \\
& =-\frac{\rho_{0 q}^{2} c^{4} \gamma_{c}^{2}}{8 \pi \varepsilon_{0} \eta^{2} \rho_{0}^{2}}\left[\frac{a}{2}+\frac{c}{4 \sqrt{4 \pi \eta \rho_{0}}} \sin \left(\frac{2 a}{c} \sqrt{4 \pi \eta \rho_{0}}\right)-\frac{c^{2}}{4 \pi \eta \rho_{0} a} \sin ^{2}\left(\frac{a}{c} \sqrt{4 \pi \eta \rho_{0}}\right)\right] \approx \\
& \approx-\frac{q^{2} \gamma_{c}^{2}}{40 \pi \varepsilon_{0} a}\left(1-\frac{3 \eta m}{7 a c^{2}}\right) \\
& \int_{r=a}^{\infty} \frac{c^{2} \varepsilon_{0}}{4} F_{\mu v} F^{\mu v} d V=-\frac{\varepsilon_{0}}{2} \int_{r=a}^{\infty} E_{o}^{2} d V= \\
& =-\frac{\rho_{0 q}^{2} c^{4} \gamma_{c}^{2}}{8 \pi \varepsilon_{0} \eta^{2} \rho_{0}^{2} a}\left[\frac{c}{\sqrt{4 \pi \eta \rho_{0}}} \sin \left(\frac{a}{c} \sqrt{4 \pi \eta \rho_{0}}\right)-a \cos \left(\frac{a}{c} \sqrt{4 \pi \eta \rho_{0}}\right)\right]^{2} \approx \\
& \approx-\frac{q^{2} \gamma_{c}^{2}}{8 \pi \varepsilon_{0} a}\left(1-\frac{3 \eta m}{5 a c^{2}}\right)
\end{aligned}
$$

\section{CONCLUSION}

In the framework of the covariant theory of gravitation, a conclusion was made for the gravitational field that the contribution into the relativistic energy of the system from the energy of the matter at rest in the gravitational scalar potential was twice as large in its absolute value as the integral of the tensor invariant of the gravitational field, and the same held true for the electromagnetic field (Fedosin, 2012b). Would it be different in case of the relativistic uniform system, where the particles of matter are not motionless, but are moving with the Lorentz factor as in Equation (2), which depends on the current radius?

To answer this question, we can sum up the integrals in Equation (12) taken with respect to the tensor invariant both inside and outside the sphere, and compare the result with Equation (11). This gives the following:

$$
\begin{aligned}
& \int_{r=0}^{a} \frac{c^{2} \varepsilon_{0}}{4} F_{\mu \nu} F^{\mu v} d V+\int_{r=a}^{\infty} \frac{c^{2} \varepsilon_{0}}{4} F_{\mu \nu} F^{\mu v} d V= \\
& \int_{r=0}^{\infty} \frac{c^{2} \varepsilon_{0}}{4} F_{\mu \nu} F^{\mu v} d V=-\frac{1}{2} \int \rho_{0 q} \varphi_{i} \gamma^{\prime} d V
\end{aligned}
$$

Thus, the relation for the energies does not depend on the type of system uniformity - both in the classical and relativistic cases, the relation remains the same. It should be noted that due to the random motion of particles the total magnetic field induction is zero everywhere, and therefore the sum of the integrals on the left side of Equation (13) is equal in its absolute value to the electric potential energy of the system.

At first glance, such a coincidence may seem occasional. Indeed, the contribution into the relativistic energy of the system from the energy of the particles in the electric field from Equation (11) is twice as large as the electric potential energy, and at $\mathbf{B}=0$ it is up to a sign equal to the double value of the integral of the tensor invariant on the left-hand side of Equation (13). However, it is generally accepted that the electromagnetic field energy is positive and is calculated by volume integration of the temporary component $W^{00}$ of the stress-energy tensor of the electromagnetic field. The field energy obtained this way differs by its sign from the integral of the tensor invariant, which is negative. Hence it follows that the terms in the relativistic energy of the system, which are responsible for the energy of the particles in the electromagnetic field and for the energy of the electromagnetic field itself, differ both from the electric potential energy and from the field energy, calculated with the help of the stress-energy tensor.

Given that the effective charge density inside the sphere is $\rho_{q}=\rho_{0 q} \gamma^{\prime}$, the two variants of estimating the electric potential energy used in electrostatics can be represented as follows: 


$$
U_{E}=\frac{1}{2} \int \rho_{0 q} \varphi_{i} \gamma^{\prime} d V=\frac{\varepsilon_{0}}{2} \int E^{2} d V
$$

The electric potential energy $U_{E}$ represents the total contribution into the relativistic energy of the system made due to the presence of the electric charges in the system. Thus, in (Feynman at al., 1964) the energy $U_{E}$ is calculated using the method of virtual work by transferring the charges from infinity to the sphere until the sphere achieves a certain radius. While this work is performed, the charges acquire energy in the electric potential of the sphere, and the energy of the field itself increases. Thus, the energy $U_{E}$ contains two components - the energy of the charges in the electric potential and the field energy.

Let us now sum up the left-hand sides of Equations (11) and (13) to find the total contribution into the relativistic energy of the system, arising due to the presence of the electric charges. If we take into account the right-hand side of Equation (13) and the definition of the electric potential energy in Equation (14), we will again obtain the energy $U_{E}$. This shows that indeed the contribution into the system's energy is made by the energy of the charges in the electric potential as well as by the field energy. However, as mentioned above, these energies coincide neither with the electric potential energy nor with the field energy, found using the stress-energy tensor of the electromagnetic field. This arises due to difference between the covariant approach that takes into account the principle of least action, and the noncovariant approach of the classical electrostatics.

Note also that Equation (13) can be interpreted as an example of action of the theorem of equipartition of energy. According to this theorem, the degrees of freedom, included in the Hamiltonian quadratically, contribute to the energy of the system two times less than the degrees of freedom, included in the Hamiltonian linearly (Huang, 1987). The electric field strength is included in the tensor invariant quadratically and the electric field potential is included in relativistic energy linearly. As a result, according to Equation (13), the field strength and the field potential can be considered separate and independent degrees of freedom of the electromagnetic field, which are equally necessary for description of the processes in the field.

\section{REFERENCES}

Fedosin, S.G., 2012a. The radius of the proton in the selfconsistent model. Hadronic Journal, 35(4):349-363.

Fedosin, S.G., 2012b. The Hamiltonian in covariant theory of gravitation. Advances in Natural Science, 5(4):5575.

Fedosin, S.G., 2014a. The integral energy-momentum 4vector and analysis of $4 / 3$ problem based on the pressure field and acceleration field. American Journal of Modern Physics, 3(4):152-167.

Fedosin, S.G., 2014b. The procedure of finding the stressenergy tensor and equations of vector field of any form. Advanced Studies in Theoretical Physics, 8(18):771-779.

Fedosin, S.G., 2015. Relativistic energy and mass in the weak field limit. Jordan Journal of Physics, 8(1):116.

Fedosin, S.G., 2016a. Estimation of the physical parameters of planets and stars in the gravitational equilibrium model. Canadian Journal of Physics, 94(4):370-379.

Fedosin, S.G., 2016b. About the cosmological constant, acceleration field, pressure field and energy. Jordan Journal of Physics, 9(1):1-30.

Fedosin, S.G., 2016c. The virial theorem and the kinetic energy of particles of a macroscopic system in the general field concept. Continuum Mechanics and Thermodynamics, 29(2):361-371.

Feynman, R., Leighton, R., Sands, M., 1964. The Feynman lectures on physics. 2: Addison Wesley.

Huang, K., 1987. Statistical mechanics (2nd ed.). John Wiley and Sons. pp. 136-138.

Kelly, J.J., 2001. Nucleon charge and magnetization densities. arXiv:hep-ph/0111251. https://arxiv.org/abs/hep-ph/0111251

Yakhshiev, U., Kim, H.C., 2013. Transverse charge densities in the nucleon in nuclear matter. Physics Letters B, 726(1-3):375-381. 